\title{
Symbolic analysis of sub-optimal bang-bang control mechanisms in base isolated structures
}

\author{
Robert Sebastianelli ${ }^{\mathrm{a}, \mathrm{b}}$ and Mark A. Austin ${ }^{\mathrm{b}, *}$ \\ ${ }^{\mathrm{a}}$ Institute for Systems Research, University of Maryland, College Park, MD 20742, USA \\ ${ }^{\mathrm{b}}$ Department of Civil and Environmental Engineering, University of Maryland, College Park, MD 20742, USA
}

Received 15 October 2007

Revised 2008

\begin{abstract}
In the performance-based design of earthquake-resistant structures, researchers have recently proposed protection systems where base isolation devices are supplemented by active control mechanisms. Established approaches to understanding this problem domain rely on numerical and experimental analyses, which have the disadvantage of obscuring potential insight into cause-and-effect relationships existing between parameters of sub-optimal control and their affect on linear and nonlinear system response. As a first step toward mitigating this limitation, this paper explores the role of symbolic analysis in understanding how sub-optimal bang-bang control mechanisms depend on design objectives and their impact on performance of base isolated structures. New results are obtained through three avenues of investigation: (1) Single- and two-degree-of-freedom systems, (2) Restricted classes of multi-degree-of-freedom systems, and (3) Sensitivity of parameters in modified bang-bang control to localized nonlinear deformations in the base isolation devices. The principle outcome is matrices of symbolic expressions for bang-bang control expressed in terms of the structural system parameters and state. We identify modeling constraints and limits (e.g., perfect isolation) where lengthy symbolic expressions simplify to the point where relationships between the inner workings of the bang-bang control strategy and specific design objectives become evident.
\end{abstract}

Keywords: Base isolation, symbolic analysis, sub-optimal bang-bang control

\section{Problem statement}

The design of structures to resist earthquake loading is complicated by the large uncertainty in predicting the spatial and temporal nature of future seismic events. Additional uncertainties exist due to the limited ability of models and computational tools to properly describe the nonlinear response under severe excitations. Consequently designers often have difficulty in making qualitative decisions regarding the adequacy of a design and in choosing rationally among design alternatives. Problems of this type are becoming prevalent because structural systems requiring special performance criteria are coming into vogue. As a case in point, the emerging interest in base isolation systems supplemented by active control is motivated by the observation that levels of structural response in both moderate and severe earthquakes can be significantly reduced by: (1) introducing mechanisms to separate the natural periods of vibration for the main structural system from the details of ground motions, and then (2) complementing the isolated system behavior with active control. Because the performance of isolated systems is controlled by displacements, versus forces in conventional structures, special recommendations for performance criteria and design procedures are needed. The recent AASHTO and ICBO design codes $[1,13]$ contain code provisions prescribing a series of standard performance levels for design, together with acceptable levels of structural and non-structural damage, and suggested methods of analysis for performance evaluation. Under minor and moderate earthquake loadings, for example, base isolated structures should suffer no structural damage. For design

\footnotetext{
*Corresponding author. E-mail: austin@umd.edu.
} 
earthquakes corresponding to the maximum credible ground motion for the site, the main structural members are expected to remain essentially elastic, with nonlinear deformations (i.e., damage) restricted to the isolation devices. Simplified methods of design for base isolated structures have been proposed by Turkington et al. [26,27], Ghobarah and Ali [9], among others. While these performance-based code provisions and simplified design procedures give high-level guidance regarding acceptable and unacceptable levels of performance (and how to achieve it), there is a mounting body of evidence that base isolation may not always provide adequate protection [10,11,31]. Under moderate ground motions, for example, design cases exist where the protection of sensitive and expensive equipment is of paramount importance. The combination of base isolation and active control holds the potential for creating so-called vibration-free environments [12]. Under severe ground motions, one concern is the possibility of localized buckling of the isolator devices and/or collapse of the structure caused by truly excessive lateral displacements of isolator elements [19]. A second area of concern is the inability of base isolation to protect structures against near-source, high-velocity, long-period pulse earthquakes, resulting in excessively large base drifts $[10,11,15,24]$.

From a design standpoint, we seek preliminary design and analysis procedures that use performance-based metrics (e.g., displacements, velocities, energy) to capture the benefits of active control and base isolation, but are not overly complicated [3]. Then, later in the design process when complete details of the structural system have been determined, detailed methods of analysis and optimization are needed for the optimal tuning of isolation and active control device parameters. The hypothesis of our work is that formulation of the appropriate methodologies will be facilitated if cause-and-effect relationships existing between design objectives, properties of the control, and their effect on the system response are known.

\section{Scope and objectives}

Bang-bang control is a strategy where force pulses are designed to counteract displacements caused by earthquake ground motions. Early research [17] indicates that significant improvements in performance are possible even when active control corresponds to force pulses applied only during the most severe intervals of system response. While numerical algorithms exist for solving the Lyapunov matrix equation, we still have a poor understanding of the underlying state-level mechanisms (displacements and velocities) that make bang-bang control work, as well as the "performance improvements" possible with active components? Several strategies for implementation seem possible: (1) use a linear control theory, but iteratively adjust the structural parameters to account for the nonlinear behavior, (2) use a nonlinear control theory, or (3) use a linear control theory that has been shown to provide effective improvements to the system response, even when nonlinearities in behavior are not explicitly captured in the system model.

To quantitatively assess these options, we have developed a computational framework for energy-balance and power-demand assessment of base isolated system supplemented by sub-optimal bang-bang control. The timehistory simulations have exposed difficulties in connecting severity of earthquake attack to predictions of maximum displacements, energy consumption, and the need to turn the active control system off after the ground motions have ceased [23]. Our observations are consistent with those of Miller and co-workers [17]. We have also shown that by looking at the phase relationship of actuator action relative to structural dynamics, simplified strategies of bang-bang control are possible [5]. The result is a simplified strategy of control that closely mirrors semi-active damping mechanisms [21].

This paper explores the role of symbolic analysis in understanding how sub-optimal bang-bang control mechanisms depend on design objectives and their impact on performance of base isolated structures. New results are obtained through three avenues of investigation:

1. Single- and two-degree-of-freedom systems. We explore the extent to which symbolic analysis can provide insight into the connection between selection of an appropriate active control synthesis method and its followthrough to a specific implementation. We will soon see that for structures having more than two-degrees of-freedom, the symbolic expressions are computationally intractable, even for Mathematica [28].

2. Restricted classes of multi-degree-of-freedom systems. Starting with relatively simple symbolic expressions for bang-bang control of a one degree of freedom structure, we determine restrictions on the structural model that allow for the basic solution to be scaled up to a $n$-DOF system. 
3. Sensitivity analysis. We explore the sensitivity of parameters in modified bang-bang control to localized nonlinear deformations in the base isolation devices.

The principle outcome of these research avenues is matrices of symbolic expressions for bang-bang control (i.e., $\mathbf{B}^{\mathrm{T}} \mathbf{S}$ ) (details given below) expressed in terms of the structural system parameters (i.e., $m, k$ and $c \ldots$ ) and state (i.e., displacements and velocities). We identify modeling constraints and limits that (e.g., perfect isolation) where lengthy symbolic expressions simplify to the point where relationships between the inner workings of the bang-bang control strategy and specific design objectives become evident.

\section{Sub-optimal bang-bang control}

In order to carry out a dynamic analysis, real structures are modeled mathematically as families of differential equations. The well known general equation of motion for a multi-degree of freedom system subject to an earthquake load and external active controlling forces is as follows:

$$
\mathbf{M} \ddot{x}(t)+\mathbf{C} \dot{x}(t)+\mathbf{K} x(t)=\mathbf{H} u(t)-\mathbf{M} r \ddot{x}_{g}(t) .
$$

In Eq. (1), $x(t)$ is a $n$-dimensional vector representing the relative displacements of the $n$ degrees of freedom. At time $t=0$, the ground velocities and displacements (i.e., $x(t)$ and $\dot{x}(t)$ ) are assumed to be zero. The initial acceleration can be computed through solutions to Eq. (1). Similarly, at some time after the ground motions have ceased, damping mechanisms will ensure that the systems displacements and velocities will return to a level that is effectively zero. $\mathbf{M}, \mathbf{C}$, and $\mathbf{K}$ are the mass, damping, and stiffness $n \times n$ matrices, respectively. $\ddot{x}_{g}(t)$ represents the earthquake ground acceleration, and $r$ is a $(n \times 1)$ vector describing movement in each of the structural degrees of freedom due to a unit ground displacement. $\mathbf{H}$ is an $n \times p$ matrix that designates the location of the controller(s). $u(t)$ is a $p$-dimensional vector that represents the control force of $p$-number of controllers. The first-order state-space form of Eq. (1) is as follows:

$$
\dot{z}(t)=\mathbf{A} z(t)+\mathbf{B} u(t)-\mathbf{W} \ddot{x}_{g}(t) .
$$

In Eq. (2), $z(t)=[x(t), \dot{x}(t)]^{T}$ and

$$
\mathbf{A}=\left[\begin{array}{cc}
\mathbf{0} & \mathbf{1} \\
-\mathbf{M}^{-\mathbf{1}} \mathbf{K}-\mathbf{M}^{-\mathbf{1}} \mathbf{C}
\end{array}\right], \quad \mathbf{B}=\left[\begin{array}{c}
\mathbf{0} \\
\mathbf{M}^{-\mathbf{1}} \mathbf{H}
\end{array}\right] \quad \text { and } \quad \mathbf{W}=\left[\begin{array}{l}
0 \\
r
\end{array}\right]
$$

\subsection{Sub-optimal bang-bang control}

The control objective for bang-bang control is minimization of the integral:

$$
J(t)=\frac{1}{2} \int_{0}^{t} z^{T}(\tau) \mathbf{Q} z(\tau) d \tau,
$$

where $\mathbf{Q}$ is a positive semi-definite matrix whose content is left for the designer to choose. A key tenet of our work is that the terms in $\mathbf{Q}$ should be selected so that the bang-bang control strategy has a well defined physical meaning (e.g., internal energy in the superstructure and/or isolation devices). The well known optimal control solution [7,29, 30] for a system in the form of Eq. (2) and which minimizes Eq. (4) is:

$$
u(t)=-U_{\max } \operatorname{sgn}\left[\mathbf{B}^{\mathbf{T}} \lambda(t)\right],
$$

where $\lambda(t)$ is known as the costate vector that is obtained by solving the following differential equation:

$$
\dot{\lambda}(t)=-\mathbf{A}^{\mathbf{T}} \lambda(t)-\mathbf{Q} z(t),
$$

and $\mathrm{U}_{\max }$ is a scalar that represents the maximum actuator control force. Numerically stable integration algorithms such as the Discrete Implicit Runge-Kutta (DIRK2) method can be used to accomplish this task. Theoretical considerations can guide the selection of initial conditions for SDOF systems. However, for all other problems 
of practical importance, solutions to Eq. (6) are complicated by a lack of theoretical guidance for choosing the differential equation's initial conditions. An incorrect assumption (on the initial conditions) will lead to a numerical solution with time-varying characteristics that are correct, but is out of phase with the "correct optimal control solution." To avoid solving Eq. (6) at each time step for the entire time history response, a suboptimal bang-bang control law has been proposed by Wu and Soong [30]. Instead of minimizing Eq. (4), the objective of suboptimal bang-bang control is to minimize the derivative of the following generalized energy function:

$$
V[z(t)]=z^{T}(t) \mathbf{S} z(t) .
$$

Equation (7) is also referred to as the Lyapunov function, where the $\mathbf{S}$ matrix is the solution to the following Lyapunov matrix equation:

$$
\mathbf{A}^{\mathbf{T}} \mathbf{S}+\mathbf{S A}=-\mathbf{Q}
$$

Taking the time derivative of Eq. (7) and substituting in the closed-loop state equation leads to the following equation results $[16,30]$ :

$$
\dot{V}[z(t)]=-z^{T}(t) \mathbf{Q} z(t)+2 u^{T}(t) \mathbf{B}^{\mathbf{T}} \mathbf{S} z(t) .
$$

Close inspection of Eq. (9) indicates that in order for this equation to be a minimum for all possible state variables, $z(t)$, the second term on the right-hand side of Eq. (9) should result in a negative scalar for all possible $z(t)$, and moreover, $u(t)$ must be set to a maximum, say $U_{\max }$. An appropriate choice for $u(t)$ that fulfills these two criteria is:

$$
u(t)=-U_{\max } \operatorname{sgn}\left[\mathbf{B}^{\mathbf{T}} \mathbf{S} z(t)\right]
$$

Substituting Eq. (10) into Eq. (1) gives:

$$
\mathbf{M} \ddot{x}(t)+\mathbf{C} \dot{x}(t)+\mathbf{K} x(t)=-\mathbf{H} U_{\max } \operatorname{sgn}\left[\mathbf{B}^{\mathbf{T}} \mathbf{S}\left(\begin{array}{c}
x(t) \\
\dot{x}(t)
\end{array}\right)\right]-\mathbf{M} r \ddot{x}_{g}(t) ;
$$

where the matrix, $\mathbf{S}$, is the $2 n \times 2 n$ matrix solution to the Lyapunov matrix equation given in Eq. (8) and $\mathbf{B}$ is a $2 n \times p$ matrix as defined by Eq. (3). The control force, $u(t)$, switches from one extreme to another (i.e., the control force is always exerting its maximum force in either the positive or negative direction). Since the control force always takes on maximum values, the full capabilities of the actuators can be exploited.

\subsection{Damping model considerations}

Damping is the dissipation or removal of energy from a structure. In spite of a large amount of research (see, for example, references $[2,14,18,22,25]$ ), our present-day understanding of damping mechanisms remains primitive. One major reason for this is that unlike the mass and stiffness properties, the matrix product $\mathbf{C} \dot{x}(t)$ is not a directly measurable quantity. Complications include the lack of understanding as to which state variables (and underpinning mechanisms/physical processes) are relevant in determining damping forces [2]. Hence, mathematical damping models are formulated under the dual objectives of: (1) enabling reasonably accurate estimates of performance, and (2) mathematical convenience.

Tsai and Kelly [25] report that since the isolation system and superstructure are made of materials with significantly different energy dissipation characteristics, overall damping of the combined system is usually non-proportional (i.e., transformation of the classical mode coordinates leads to equations that are coupled). Designers naturally want to know if approximations to the damping model, such as simply ignoring the off-diagonal terms in the damping matrix, can be made? Tsai and Kelly conclude that the effects of non-proportional damping on secondary systems (e.g., light equipment) can be significant, but otherwise, sufficient accuracy can be obtained through the use of standard/simpler damping models. This result is particularly important for the preliminary stages of design. Moreover, since the linear and nonlinear structural dynamics of isolated systems is dominated by first and second mode vibrations, damping matrices are often assumed to take the form:

$$
\mathbf{C}=\alpha \mathbf{M}+\beta \mathbf{K},
$$


where $\alpha$ and $\beta$ are constants [18,22]. This is Rayleigh or proportional damping. Rayleigh damping is popular because it only requires the use of existing (i.e., the mass and stiffness) matrices. Under moderate seismic attack, where both the superstructure and isolation system are expected to remain linearly elastic, Eq. (12) will provide a reasonable approximation to actual damping. Under severe seismic attack, the base isolation system will deform well into the inelastic range, leaving the main structural system to remain essentially elastic. Studies indicate that if $\mathbf{C}$ is based on initial elastic stiffness, then excursions into the inelastic range result in damping that decreases during the response - this observation is not consistent with notions that damping should increase with incipient plasticity. Moss and co-workers [18] point out, however, that more realistic results can be obtained with Rayleigh damping models based on tangent stiffness and by using the the first and a higher (not the second) mode of vibration in the computation of $\alpha$ and $\beta$.

\section{Symbolic analysis for 1-DOF system}

A key shortcoming in using numerical analysis packages to solve the Lyapunov equation for matrix $\mathbf{S}$, followed by the computation of $\mathbf{B}^{\mathbf{T}} \mathbf{S} z(t)$, is that any potential insight into the appropriate cause-and-effect relationships is buried inside the numerical procedure. To mitigate this shortcoming, we explore the extent to which symbolic analysis procedures can provide insight into the connection between an appropriate selection of $\mathbf{Q}$, positioning of

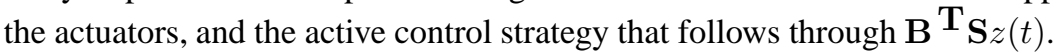

\subsection{Symbolic representation for 1-DOF bang-bang control strategy}

Consider a 1-DOF system with stiffness, $k$, mass, $m$, and without any loss of generality, damping, $c=\alpha \cdot m+\beta \cdot k$. Assume that the 1-DOF system has an actuator acting on the DOF. For the following general choice of $\mathbf{Q}$,

$$
\mathbf{Q}=\left[\begin{array}{rr}
k^{\star} & 0 \\
0 & 0
\end{array}\right]
$$

where $k^{\star}$ is a real, positive number, the symbolic representation for the $\mathbf{B}^{\mathbf{T}} \mathbf{S}$ matrix product is:

$$
\mathbf{B}^{\mathbf{T}} \mathbf{S}=\left[\begin{array}{ll}
0 & \frac{1}{m}
\end{array}\right]\left[\begin{array}{cc}
\frac{m k^{\star}}{2(\alpha \cdot m+\beta \cdot k)}+\frac{(\alpha \cdot m+\beta \cdot k) k^{\star}}{2 k} & \frac{m k^{\star}}{2 k} \\
\frac{m k^{\star}}{2 k} & \frac{m^{2} k^{\star}}{2 k(\alpha \cdot m+\beta \cdot k)}
\end{array}\right]=\left[\frac{k^{\star}}{2 k} \frac{m k^{\star}}{2 k(\alpha \cdot m+\beta \cdot k)}\right] .
$$

When the terms in $\mathbf{Q}$ are selected to minimize potential energy in the 1-DOF system (i.e., $k^{\star}=k$ ), Eq. (14) simplifies to:

$$
\mathbf{B}^{\mathbf{T}} \mathbf{S}=\left[0 \frac{1}{m}\right]\left[\begin{array}{cc}
\frac{m k}{2(\alpha \cdot m+\beta \cdot k)}+\frac{\alpha \cdot m+\beta \cdot k}{2} & \frac{m}{2} \\
\frac{m}{2} & \frac{m^{2}}{2(\alpha \cdot m+\beta \cdot k)}
\end{array}\right]=\left[\frac{1}{2} \frac{m}{2(\alpha \cdot m+\beta \cdot k)}\right] .
$$

Similarly, when

$$
\mathbf{Q}=\left[\begin{array}{cc}
0 & 0 \\
0 & m^{\star}
\end{array}\right]
$$

where $m^{\star}$ is a real, positive number, the $\mathbf{B}^{\mathbf{T}} \mathbf{S}$ matrix product evaluates to:

$$
\mathbf{B}^{\mathbf{T}} \mathbf{S}=\left[0 \frac{1}{m}\right]\left[\begin{array}{cc}
\frac{m^{\star} k}{2(\alpha \cdot m+\beta \cdot k)} & 0 \\
0 & \frac{m m^{\star}}{2(\alpha \cdot m+\beta \cdot k)}
\end{array}\right]=\left[0 \frac{m^{\star}}{2(\alpha \cdot m+\beta \cdot k)}\right]
$$

If the kinetic energy, $T(\dot{x}(t))$, in this 1-DOF system is minimized, i.e., $m^{\star}=m$, Eq. (17) becomes:

$$
\mathbf{B}^{\mathbf{T}} \mathbf{S}=\left[0 \frac{1}{m}\right]\left[\begin{array}{cc}
\frac{m k}{2(\alpha \cdot m+\beta \cdot k)} & 0 \\
0 & \frac{m^{2}}{2(\alpha \cdot m+\beta \cdot k)}
\end{array}\right]=\left[0 \frac{m}{2(\alpha \cdot m+\beta \cdot k)}\right] .
$$




\section{Actuators applied to structural dof.}

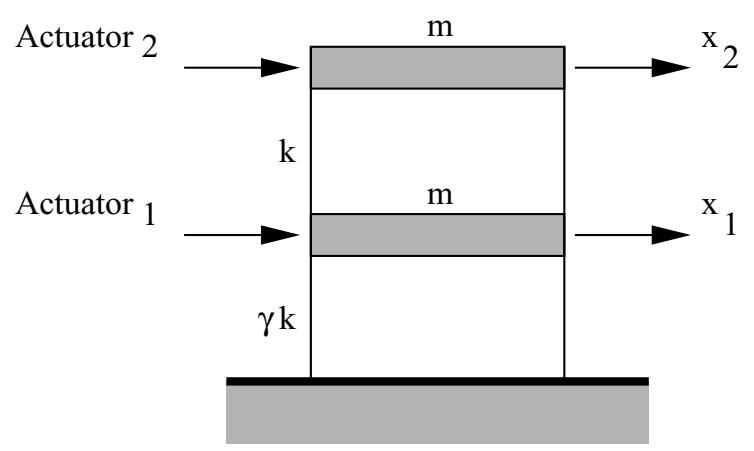

\section{Base-level and interstorey actuators.}

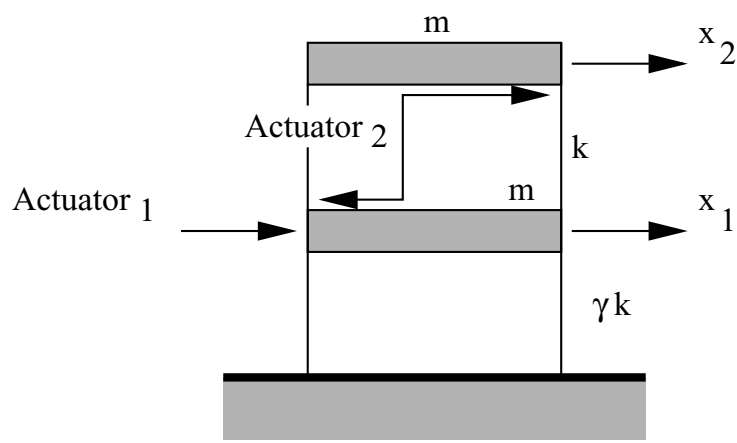

Fig. 1. 2-DOF System Configurations. Left: Actuators applied at the structural degrees of freedom. Right: One actuator is applied to the lower floor dof. A second actuator is applied between the floor levels.

\subsection{Linear properties of the lyapunov matrix equation}

The multi-objective design of bang-bang control strategies is simplified by noting that for a given linear system, matrix $\mathbf{A}$ is fixed and matrix $\mathbf{S}$ is linearly dependent on $\mathbf{Q}$. In other words, given two Lyapunov matrix equations:

$$
\mathbf{A}^{\mathbf{T}} \mathbf{S}_{1}+\mathbf{S}_{1} \mathbf{A}=\mathbf{Q}_{1} \text { and } \mathbf{A}^{\mathbf{T}} \mathbf{S}_{\mathbf{2}}+\mathbf{S}_{\mathbf{2}} \mathbf{A}=\mathbf{Q}_{\mathbf{2}},
$$

linearity of the Lyapunov matrix equations with respect to $\mathbf{S}$ implies:

$$
\mathbf{A}^{\mathbf{T}}\left(a \mathbf{S}_{\mathbf{1}}+b \mathbf{S}_{\mathbf{2}}\right)+\left(a \mathbf{S}_{\mathbf{1}}+b \mathbf{S}_{\mathbf{2}}\right) \mathbf{A}=a \mathbf{Q}_{\mathbf{1}}+b \mathbf{Q}_{\mathbf{2}} .
$$

where $a$ and $b$ are arbitrary coefficients. Hence, in the Lyapunov matrix equation, the $\mathbf{B}^{\mathbf{T}} \mathbf{S}$ matrix product that minimizes the total (potential + kinetic) energy of this 1-DOF system is the sum of Eqs (15) and (18).

\section{Symbolic analysis for a 2-DOF system}

The left- and right-hand sides of Fig. 1 show the two configurations of actuator placement that will be considered in this study. For the case where actuators are placed directly at the structural degrees of freedom,

$$
H=\left[\begin{array}{ll}
1 & 0 \\
0 & 1
\end{array}\right] \text {. }
$$

A more likely configuration is placement of an actuator at the base and a second actuator between the first and second floors. In this case,

$$
H=\left[\begin{array}{cc}
1 & 0 \\
-1 & 1
\end{array}\right]
$$

\subsection{Symbolic analysis}

Research $[4,6]$ suggests that overall "blanket minimization" of structure-level energy (i.e., potential and kinetic energy) is an overly simplified view of desirable behavior. Instead, analysis procedures should allow for potential energy terms in the system superstructure to be considered separately from potential energy in the base isolation devices. For the 2-DOF systems in Fig. 1, a suitable form for $\mathbf{Q}$ is:

$$
\mathbf{Q}=\left[\begin{array}{cccc}
a \gamma k+b k & -b k & 0 & 0 \\
-b k & b k & 0 & 0 \\
0 & 0 & 0 & 0 \\
0 & 0 & 0 & 0
\end{array}\right]
$$


The parameter setting ( $a=1, b=0)$ corresponds to minimization of potential energy in the isolation device alone. Conversely, the parameter setting $(a=0, b=1)$ corresponds to minimization of potential energy in the superstructure alone. Assuming that system damping follows the form $\mathbf{C}=\alpha \cdot \mathbf{M}+\beta \cdot \mathbf{K}$, then the symbolic solution to $\mathbf{A}^{\mathbf{T}} \mathbf{S}+\mathbf{S A}=-\mathbf{Q}$, as determined by Mathematica, is as follows:

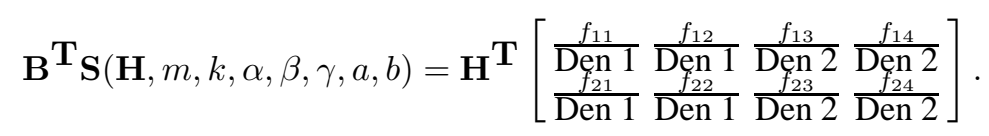

Terms in the denominator are:

$$
\begin{aligned}
\text { Den } 1= & 2\left(2 \beta^{2} \gamma(2+\gamma) k^{2}+\alpha \beta\left(4+8 \gamma+\gamma^{2}\right) k m+m\left(\left(4+\gamma^{2}\right) k+2 \alpha^{2}(2+\gamma) m\right)\right), \\
\text { Den } 2= & 2\left(\beta^{2} \gamma k^{2}+\alpha \beta(2+\gamma) k m+\alpha^{2} m^{2}\right)\left(2 \beta^{2} \gamma(2+\gamma) k^{2}+\alpha \beta\left(4+8 \gamma+\gamma^{2}\right) k m\right. \\
& \left.+m\left(\left(4+\gamma^{2}\right) k+2 \alpha^{2}(2+\gamma) m\right)\right) .
\end{aligned}
$$

And terms in the numerator are:

$$
\begin{aligned}
& f_{11}=b m\left((1+\alpha \beta)(2+\gamma) k+2 \alpha^{2} m\right)+a\left(2 \beta^{2} \gamma(2+\gamma) k^{2}\right. \\
& \left.+\alpha \beta\left(2+7 \gamma+\gamma^{2}\right) k m+m\left(\left(2-\gamma+\gamma^{2}\right) k+2 \alpha^{2}(1+\gamma) m\right)\right), \\
& f_{12}=(a-b) m\left((1+\alpha \beta)(2+\gamma) k+2 \alpha^{2} m\right) \\
& =-b m\left((1+\alpha \beta)(2+\gamma) k+2 \alpha^{2} m\right)+a m\left((1+\alpha \beta)(2+\gamma) k+2 \alpha^{2} m\right) \text {, } \\
& f_{13}=m\left(b m\left(\alpha m\left((2+\gamma) k+2 \alpha^{2} m\right)+\beta k\left(2 \gamma k+2 \alpha^{2} m+\alpha^{2} \gamma m\right)\right)\right. \\
& +a\left(2 \beta^{3} \gamma(2+\gamma) k^{3}+\alpha \beta^{2}\left(4+12 \gamma+3 \gamma^{2}\right) k^{2} m+\alpha m^{2}\left(\left(2-\gamma+\gamma^{2}\right) k\right.\right. \\
& \left.\left.\left.\left.+2 \alpha^{2}(1+\gamma) m\right)+\beta k m\left(\left(4-2 \gamma+\gamma^{2}\right) k+\alpha^{2}\left(6+9 \gamma+\gamma^{2}\right) m\right)\right)\right)\right), \\
& f_{14}=m\left(-b m\left(-\beta \gamma^{2} k^{2}+\alpha^{2} \beta(2+3 \gamma) k m+2 \alpha^{3} m^{2}\right.\right. \\
& \left.+\alpha k\left(\beta^{2} \gamma(2+\gamma) k-(-2+\gamma) m\right)\right)+a\left(2 \beta^{3} \gamma(2+\gamma) k^{3}\right. \\
& +2 \alpha \beta^{2}\left(2+5 \gamma+\gamma^{2}\right) k^{2} m+\alpha m^{2}\left(-(-2+\gamma) k+2 \alpha^{2} m\right) \\
& \left.\left.\left.+\beta k m\left(4 k+\alpha^{2}(6+5 \gamma) m\right)\right)\right)\right) \text {, } \\
& f_{21}=(a-b)\left(2 \beta^{2} \gamma(2+\gamma) k^{2}+\alpha \beta(2+5 \gamma) k m+m\left((2-3 \gamma) k+2 \alpha^{2} m\right)\right) \\
& =-b\left(2 \beta^{2} \gamma(2+\gamma) k^{2}+\alpha \beta(2+5 \gamma) k m+m\left((2-3 \gamma) k+2 \alpha^{2} m\right)\right) \\
& +a\left(2 \beta^{2} \gamma(2+\gamma) k^{2}+\alpha \beta(2+5 \gamma) k m+m\left((2-3 \gamma) k+2 \alpha^{2} m\right)\right), \\
& f_{22}=a m\left((1+\alpha \beta)(2+\gamma) k+2 \alpha^{2} m\right)+b\left(2 \beta^{2} \gamma(2+\gamma) k^{2}+\alpha \beta\left(2+7 \gamma+\gamma^{2}\right) k m\right. \\
& \left.+m\left(\left(2-\gamma+\gamma^{2}\right) k+2 \alpha^{2}(1+\gamma) m\right)\right), \\
& f_{23}=f_{14}, \\
& f_{24}=m\left(b \left(2 \beta^{3} \gamma^{2}(2+\gamma) k^{3}+\alpha \beta^{2} \gamma\left(8+10 \gamma+\gamma^{2}\right) k^{2} m+\beta k m\left(2 \gamma k+\gamma^{3} k+2 \alpha^{2} m\right.\right.\right. \\
& \left.\left.+11 \alpha^{2} \gamma m+3 \alpha^{2} \gamma^{2} m\right)+\alpha m^{2}\left(\left(2-\gamma+\gamma^{2}\right) k+2 \alpha^{2}(1+\gamma) m\right)\right)+a\left(2 \beta^{3} \gamma(2+\gamma) k^{3}\right. \\
& \left.\left.+\alpha \beta^{2}\left(4+8 \gamma+\gamma^{2}\right) k^{2} m+\alpha m^{2}\left((2+\gamma) k+2 \alpha^{2} m\right)+\beta k m\left(\left(4+2 \gamma+\gamma^{2}\right) k+3 \alpha^{2}(2+\gamma) m\right)\right)\right) \text {. }
\end{aligned}
$$

\subsection{Special cases}

The general symbolic solutions are not readily amenable to interpretation. We have found, however, that under a number of circumstances, the lengthy symbolic expressions simplify significantly. First, when $\mathbf{Q}$ contains the structural stiffness matrix in the upper-left quadrant (i.e., $a=b=1$ ), $\mathbf{B}^{\mathbf{T}} \mathbf{S}$ simplifies to: 


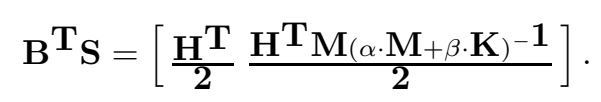

This result is consistent with the $n$-DOF model derived in the next section. The second special case occurs for structures that are perfectly isolated (i.e., $\gamma=0$ ). The symbolic expressions simply to:

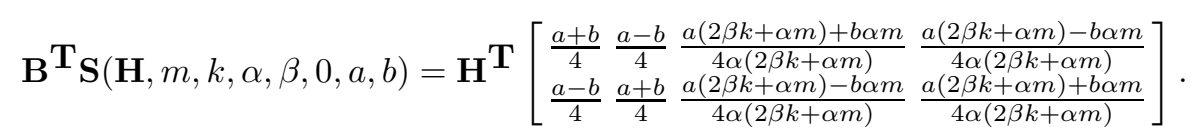

Two special cases exist with respect to Eq. 26. When $a=b=1$ (i.e., minimization of total energy), Eq. (26) simplifies further:

$$
\mathbf{B}^{\mathbf{T}} \mathbf{S}(\mathbf{H}, m, k, \alpha, \beta, 0,1,1)=\mathbf{H}^{\mathbf{T}}\left[\begin{array}{lll}
\frac{1}{2} & 0 \frac{\beta k+\alpha m}{2 \alpha(2 \beta k+\alpha m)} & \frac{\beta k}{2 \alpha(2 \beta k+\alpha m)} \\
0 & \frac{1}{2} \frac{\beta k+\alpha k+\alpha m)}{2 \alpha(2 \beta k+\alpha m)} & \frac{\beta k+\alpha m}{2 \alpha(2 \beta k+\alpha m)}
\end{array}\right] .
$$

Notice that symbolic expressions for the coefficient denominators will be non-zero as long as $\alpha \neq 0$ (here we assume that $m$ and $k$ will never be zero) and hence, generally, the bang-bang control strategy is well defined even for structures that are perfectly isolated. The second special case occurs for $a=0$ and $b=1$ (i.e., we want to minimize energy within the superstructure alone). Now Eq. (26) simplifies to:

$$
\mathbf{B}^{\mathbf{T}} \mathbf{S}(\mathbf{H}, m, k, \alpha, \beta, 0,0,1)=\mathbf{H}^{\mathbf{T}}\left[\begin{array}{cccc}
\frac{1}{4} & -\frac{1}{4} & \frac{m}{2(2 \beta k+\alpha m)} & -\frac{m}{2(2 \beta k+\alpha m)} \\
-\frac{1}{4} & \frac{1}{4} & -\frac{m}{2(2 \beta k+\alpha m)} & \frac{m}{2(2 \beta k+\alpha m)}
\end{array}\right] .
$$

In a typical base isolated structure, the time history response will be dominated by the first mode of vibration (i.e., $x_{1}(\mathrm{t}) \approx x_{2}(\mathrm{t})$ and $\dot{x}_{1}(\mathrm{t}) \approx \dot{x}_{2}(\mathrm{t})$ ). We observe that displacement and velocity pairs of this type will have little influence on the control strategy. Rather, actuator actions will be determined primarily by second- and higher-mode displacements and velocities, with the influence of velocity measurements increasing as damping approaches zero. Also notice that the row 1 elements are exactly the opposite of the row 2 elements. In other words, in the idealized case of perfect isolation, the actuator actions will work to cancel each other out.

\subsection{Effect of actuator configuration under conditions of perfect isolation}

Recall that the purpose of $\mathbf{H}$ is to map the actuator configurations to the structural degrees of freedom. The trivial case occurs when actuator forces are applied directly to each structural degree of freedom. Equation (26) simplifies to

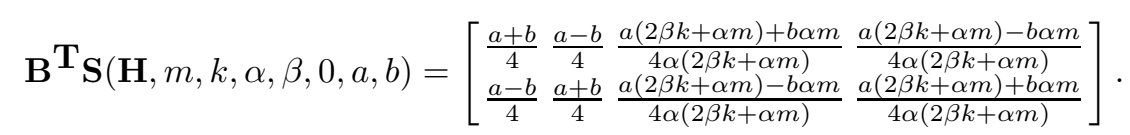

When $\mathbf{H}$ corresponds to Eq. (22) (i.e., actuator placement is as on the right-hand side of Fig. 1), Eq. (26) simplifies to,

$$
\mathbf{B}^{\mathbf{T}} \mathbf{S}(\mathbf{H}, m, k, \alpha, \beta, 0, a, b)=\left[\begin{array}{cccc}
\frac{b}{2} & -\frac{b}{2} & \frac{b m}{2(2 \beta k+\alpha m)} & -\frac{b m}{2(2 \beta k+\alpha m)} \\
\frac{a-b}{4} & \frac{a+b}{4} & \frac{a(2 \beta k+\alpha m)-b \alpha m}{4 \alpha(2 \beta k+\alpha m)} & \frac{a(2 \beta k+\alpha m)+b \alpha m}{4 \alpha(2 \beta k+\alpha m)}
\end{array}\right] .
$$

Notice that the second row of $\mathbf{B}^{\mathbf{T}} \mathbf{S}$ remains unchanged. Now when $a=b=1$, Eq. (30) simplifies to:

$$
\mathbf{B}^{\mathbf{T}} \mathbf{S}(\mathbf{H}, m, k, \alpha, \beta, 0,1,1)=\left[\begin{array}{cccc}
\frac{1}{2}-\frac{1}{2} & \frac{m}{2(2 \beta k+\alpha m)} & -\frac{m}{2(2 \beta k+\alpha m)} \\
0 & \frac{1}{2} & \frac{\beta k}{2 \alpha(2 \beta k+\alpha m)} & \frac{(\beta k+\alpha m)}{2 \alpha(2 \beta k+\alpha m)}
\end{array}\right] .
$$

In other words, control of actuator 2 will be influenced by the displacement at dof 2 and a combination of velocity measurements at dof 1 and dof 2 . The control strategy for actuator 1 will be determined from difference in displacements and velocities at dof's 1 and 2. The latter observation also suggests that for systems containing more than two degrees of freedom, the forces in interstorey actuators will be determined primarily from relative displacements and velocities in the higher modes.

Similarly, when $a=0$ and $b=1$ (i.e., minimize energy within the superstructure alone), Eq. (30) simplifies to: 


$$
\mathbf{B}^{\mathbf{T}} \mathbf{S}(\mathbf{H}, m, k, \alpha, \beta, 0,0,1)=\left[\begin{array}{cccc}
\frac{1}{2} & -\frac{1}{2} & \frac{m}{(2 \beta k+\alpha m)} & -\frac{m}{(2 \beta k+\alpha m)} \\
-\frac{1}{4} & \frac{1}{4} & -\frac{m}{2(2 \beta k+\alpha m)} & \frac{m}{2(2 \beta k+\alpha m)}
\end{array}\right] .
$$

Now the control forces for both actuators are determined by differences in displacement and velocity measurements at dof 1 and dof 2 . The same observation applied to Eq. (28). Also notice that the terms of row 1 are -2 times those in row 2. Since, it is only the sign of the matrix product in $\mathbf{B}^{\mathbf{T}} \mathbf{S z}$ that matters, under conditions of perfect isolation, the control strategies for actuator 1 and 2 are 180 degrees out of phase (i.e., the actuator forces work to cancel each other out).

One surprising result from this analysis is the role of coefficient "a." From Eq. (23) one might initially think that if $\gamma$ equals zero (i.e., perfect isolation), then the value "a" doesn't matter. But Eqs (26) through (32) indicate that, clearly, this isn't the case. In other words, the symbolic analysis validates the purpose of each parameter and its role in affecting the details of structural control.

\subsection{Minimizing kinetic energy}

This exercise can be repeated for minimization of kinetic energy in the base isolator and superstructure. For the 2-DOF mass-spring-damper system shown in Fig. 1, a suitable form for $\mathbf{Q}$ is:

$$
\mathbf{Q}=\left[\begin{array}{cccc}
0 & 0 & 0 & 0 \\
0 & 0 & 0 & 0 \\
0 & 0 & c m & 0 \\
0 & 0 & 0 & d m
\end{array}\right]
$$

The symbols $c$ and $d$ in Eq. (33) represent relative amount of weight a designer places on minimizing kinetic energy in the first and second stories of the structure, respectively. When $c=d=1$ the symbolic representation of $\mathbf{B} \mathbf{T}_{\mathbf{S}}$ simplifies to:

$$
\mathbf{B}^{\mathbf{T}} \mathbf{S}=\left[\mathbf{0} \frac{\mathbf{H}^{\mathbf{T}} \mathbf{M}_{\left(\alpha \cdot \mathbf{M}_{+\beta} \cdot \mathbf{K}\right)^{-1}}}{2}\right]
$$

Notice that because solutions to the Lyapunov matrix equation are linear with respect to matrix $\mathbf{S}$, the $\mathbf{B}^{\mathbf{T}} \mathbf{S}$ matrix product that minimizes the total (potential + kinetic) is simply the sum of Eqs (25) and (34).

\section{Symbolic analysis of a n-DOF system}

When we first obtained symbolic expressions for the matrix elements in $\mathbf{B}^{\mathbf{T}} \mathbf{S}$, it was not immediately evident that when $a=b=1$ and $c=d=1$, the lengthy formulae would simplify to the formats shown in Eqs (25) and (34). This surprising result made us think about other possibilities. Specifically, starting with relatively simple expressions for solutions to $\mathbf{B}^{\mathbf{T}} \mathbf{S}$ in a one degree of freedom structure, we wondered if it would it be possible perhaps under certain restrictions - to scale this solution up to a N-DOF system? This pathway of investigation has two key benefits. Unlike numerical procedures for solution to the Lyapunov equation, symbolic expressions provide physical insight into the inner workings of the bang-bang control strategy. And second, when the matrix restrictions apply, symbolic expressions provide a tractable alternative to numerical solutions to the Lyapunov matrix equation. Here, we simply present a summary of the key results and observations.

\subsection{Minimizing potential energy}

To minimize the potential energy in a $n$-DOF system, an appropriate choice for the $\mathbf{Q}$ matrix would be:

$$
\mathbf{Q}=\left[\begin{array}{ll}
\mathbf{K} & 0 \\
\mathbf{0} & \mathbf{0}
\end{array}\right]
$$

where $\mathbf{K}$ is the $n \times n$ structural stiffness matrix and $\mathbf{0}$ is a $n \times n$ matrix of zeros. Substituting Eq. (35) into the Lyapunov matrix equation shown in Eq. (8) and solving results in the following $\mathbf{B}^{\mathrm{T}} \mathbf{S}$ matrix product: 


$$
\mathbf{B}^{\mathbf{T}} \mathbf{S}=\left[\frac{\mathbf{H}^{\mathbf{T}}}{\mathbf{2}} \frac{\left.\mathbf{H}^{\mathbf{T}} \mathbf{M}_{(\alpha \cdot \mathbf{M}} \mathbf{M}_{+\beta} \mathbf{K}\right)^{-1}}{2}\right]
$$

where $\mathbf{H}$ is a $n$ by $p$ matrix that designates the location of the controller(s). Appendix 1 shows the derivation of Eq. (36). Equation (36) holds when: (1) the mass matrix $\mathbf{M}$ is diagonal and uniform (i.e., $m_{1}=m_{2}=\ldots=m_{n}$ ), (2) linear viscous damping is present, and (3) the structural stiffness matrix $\mathbf{K}$ is well-conditioned. If the damping matrix sum $\alpha \cdot \mathbf{M}+\beta \cdot \mathbf{K}$ becomes rank deficient, then a unique solution to $\mathbf{B}^{\mathbf{T}} \mathbf{S}$ does not exist.

\subsection{Remark}

By substituting Eq. (36) into Eq. (9), the system parameters that are being minimized by the modified bang-bang control objective results:

$$
\dot{V}[x(t), \dot{x}(t)]=-x^{T}(t) \mathbf{K} x(t)+u^{T}(t) \mathbf{H}^{\mathbf{T}}\left[\mathbf{I} x(t)+\mathbf{M}(\alpha \cdot \mathbf{M}+\beta \cdot \mathbf{K})^{-1} \dot{x}(t)\right] .
$$

The first term on the right-hand side of Eq. (37) is an energy term that corresponds to double the amount of potential energy in the system at any time, $t$. Physical considerations dictate that the second term on the right-hand side of Eq. (37) must also be in terms of energy. Since the actuator force, $u(t)$, is present in Eq. (37), this term may be thought of as being made up of displacement and velocity terms that account for work that is done by the actuator force(s) at any time, $t$.

\subsection{Minimizing kinetic energy}

Minimizing the squares of the velocities of a system response may not seem like an important parameter, but internal non-structural damage (e.g., to internal walls, plumbing, etc.) is correlated to peak velocities within a structure [20]. An appropriate choice for $\mathbf{Q}$ is as follows:

$$
\mathbf{Q}=\left[\begin{array}{ll}
\mathbf{0} & \mathbf{0} \\
\mathbf{0} & \mathbf{M}
\end{array}\right]
$$

where $\mathbf{M}$ is the $n$ by $n$ structural mass matrix and $\mathbf{0}$ is a $n$ by $n$ matrix of zeros. Substituting Eq. (38) into the Lyapunov matrix equation results in the $\mathbf{B}^{\mathrm{T}} \mathbf{S}$ matrix product:

$$
\mathbf{B}^{\mathbf{T}} \mathbf{S}=\left[\mathbf{0} \frac{\mathbf{H}^{\mathbf{T}} \mathbf{M}_{\left(\alpha \cdot \mathbf{M}_{+\beta} \cdot \mathbf{K}\right)^{-1}}}{2}\right] \text {. }
$$

In Eq. (39), $\mathbf{0}$ is a $p$ by $n$ matrix of zeros and $\mathbf{H}$ is a $n$ by $p$ matrix that designates the location of the controller(s).

\subsection{Minimizing total (potential + kinetic) energy}

The total energy in an $n$-DOF system can be minimized by setting $\mathbf{Q}$ to:

$$
\mathbf{Q}=\left[\begin{array}{cc}
\mathbf{K} & \mathbf{0} \\
\mathbf{0} & \mathbf{M}
\end{array}\right] \text {. }
$$

Solutions to the Lyapunov equation corresponding to Eq. (40) are given by the sum of Eqs (35) and (38). In other words,

$$
\mathbf{B}^{\mathbf{T}} \mathbf{S}=\left[\frac{\mathbf{H}^{\mathbf{T}}}{\mathbf{2}} \mathbf{H}^{\mathbf{T}} \mathbf{M}(\alpha \cdot \mathbf{M}+\beta \cdot \mathbf{K})^{-\mathbf{1}}\right] .
$$

\section{Sensitivity to nonlinear deformations}

Now that closed form expressions for sub-optimal bang-bang control in place, we can explore sensitivity of the parameters in modified bang-bang control to localized nonlinear deformations in the base isolation devices. In a typical base isolated structure, the initial stiffness of the base isolators will be 10-20\% of the stiffness of elements in the superstructure. After the isolators have yielded, the tangent stiffness may drop to 2-10\% of elements in the superstructure, and as pointed out by Moss and co-workers [18], the damping matrix should be adjusted to use the tangent stiffness. Thus, from a research perspective the question of interest is as follows: do these nonlinearities have a significant impact on the control strategy that should be employed? We address this concern by conducting a symbolic analysis of the control strategy for minimization of potential energy alone. 


\subsection{Case study problem}

The case study problem is the 2-DOF system shown on the left-hand side of Fig. 1. Equation (36) gives:

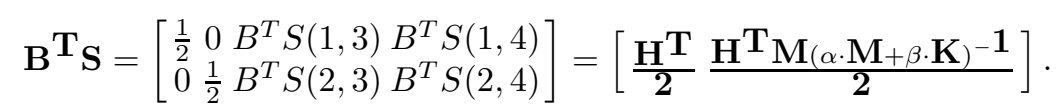

In the bang-bang control strategy, coefficients on the left- and right-hand sides of $\mathbf{B} \mathbf{T}_{\mathbf{S}}$ are multiplied by the system displacements and velocities, respectively. The terms $B^{T} S(1,3)$ through $B^{T} S(2,4)$ are elements of the matrix product $\mathbf{H}^{\mathbf{T}} \mathbf{M}(\alpha \cdot \mathbf{M}+\beta \cdot \mathbf{K})^{-\mathbf{1}} / 2$. Due to symmetry of the mass and stiffness matrices, $B^{T} S(2,3)=B^{T} S(1,4)$. Our research goal is to identify conditions in the problem formulation where coefficients for the system velocities will be either very large or, conversely, very small, compared to the displacement coefficients. The former condition will lead to control strategies heavily influenced by system velocities. The latter will lead to control strategies dominated by system displacements.

\subsection{Symbolic expressions for velocity components of bang-bang control}

Let us assume that damping is present in the form $\alpha \cdot \mathbf{M}+\beta \cdot \mathbf{K}$ such that there is percentage, $\xi$, of critical damping of the first two modes of vibration. Since detailed information on the variation of damping with frequency is seldom available, it is common practice for analysis procedures to assume equal damping ratios to both frequencies [8] (i.e., $\xi=\xi_{1} \approx \xi_{2}$ where $\xi_{1}$ and $\xi_{2}$ represent the damping ratios for the first two modes of vibration, respectively). The damping matrix coefficients $\alpha$ and $\beta$ are as follows:

$$
\alpha=\frac{2 \xi \omega_{2} \omega_{1}}{\omega_{2}+\omega_{1}} \quad \text { and } \quad \beta=\frac{2 \xi}{\omega_{2}+\omega_{1}},
$$

where $\omega_{1}$ and $\omega_{2}$ are the first and second natural frequencies of the system, respectively. From eigenvalue analysis, the natural circular frequencies of vibration are:

$$
\omega_{1}(\gamma, m, k)=\sqrt{\frac{k\left(\gamma+2-\sqrt{\gamma^{2}+4}\right)}{2 m}}
$$

and

$$
\omega_{2}(\gamma, m, k)=\sqrt{\frac{k\left(\gamma+2+\sqrt{\gamma^{2}+4}\right)}{2 m}} .
$$

Symbolic expressions for $B^{T} S(1,3), B^{T} S(1,4)$ and $B^{T} S(2,4)$ in terms of $\xi, \gamma$, and the simplifying notation $\tau=m / k$ (units of seconds ${ }^{2}$ ) are as follows:

$$
\begin{aligned}
& B^{T} S(1,3)=\left[\frac{\sqrt{\tau / 8}\left[\sqrt{(\gamma+2)-\sqrt{\gamma^{2}+4}}+\sqrt{(\gamma+2)+\sqrt{\gamma^{2}+4}}\right](1+\sqrt{\gamma})}{\sqrt{\gamma} \cdot[4+2 \gamma+4 \sqrt{\gamma}] \cdot \xi}\right] \\
& B^{T} S(1,4)=\left[\frac{\sqrt{\tau / 8}\left[\sqrt{(\gamma+2)-\sqrt{\gamma^{2}+4}}+\sqrt{(\gamma+2)+\sqrt{\gamma^{2}+4}}\right]}{\sqrt{\gamma} \cdot[4+2 \gamma+4 \sqrt{\gamma}] \cdot \xi}\right] \\
& B^{T} S(2,4)=\left[\frac{\sqrt{\tau / 8}\left[\sqrt{(\gamma+2)-\sqrt{\gamma^{2}+4}}+\sqrt{(\gamma+2)+\sqrt{\gamma^{2}+4}}\right](1+\gamma+\sqrt{\gamma})}{\sqrt{\gamma} \cdot[4+2 \gamma+4 \sqrt{\gamma}] \cdot \xi}\right]
\end{aligned}
$$


Table 1

First and second natural periods of vibration (i.e. $T_{1}$ and $T_{2}$ ) versus $\tau$ and $\gamma$

\begin{tabular}{|c|c|c|c|c|c|c|}
\hline$\tau=0.0001\left(\sec ^{2}\right)$ & $\gamma=0.001$ & $\gamma=0.005$ & $\gamma=0.01$ & $\gamma=0.05$ & $\gamma=0.10$ & $\gamma=0.15$ \\
\hline$T_{1}(\mathrm{sec})$ & 2.8103 & 1.2574 & 0.8897 & 0.3999 & 0.2846 & 0.2338 \\
\hline$T_{2}(\mathrm{sec})$ & 0.0444 & 0.0444 & 0.0444 & 0.0442 & 0.0439 & 0.0436 \\
\hline \multicolumn{7}{|l|}{$\tau=0.0010\left(\mathrm{sec}^{2}\right)$} \\
\hline$T_{1}(\mathrm{sec})$ & 8.8869 & 3.9763 & 2.8134 & 1.2646 & 0.8999 & 0.7395 \\
\hline$T_{2}(\mathrm{sec})$ & 0.1405 & 0.1404 & 0.1403 & 0.1396 & 0.1387 & 0.1378 \\
\hline \multicolumn{7}{|l|}{$\tau=0.010\left(\mathrm{sec}^{2}\right)$} \\
\hline$T_{1}(\mathrm{sec})$ & 28.1028 & 12.5742 & 8.8969 & 3.9989 & 2.8457 & 2.3385 \\
\hline$T_{2}(\mathrm{sec})$ & 0.4442 & 0.4440 & 0.4437 & 0.4415 & 0.4387 & 0.4359 \\
\hline
\end{tabular}

\subsection{Remark}

We need to make sure that the parameter values $\tau$ and $\gamma$ are selected in such way that the natural periods of vibration are representative of systems that occur in practice. The derivation is straightforward. From Eqs (44) and (45) we obtain:

$$
T_{1}=\frac{2 \pi}{w_{1}}=2 \pi \sqrt{\frac{2 \tau}{\left(\gamma+2-\sqrt{\gamma^{2}+4}\right)}}
$$

and

$$
T_{2}=\frac{2 \pi}{w_{2}}=2 \pi \sqrt{\frac{2 \tau}{\left(\gamma+2+\sqrt{\gamma^{2}+4}\right)}}
$$

Table 1 summarizes the first and second natural periods of vibration (i.e., $T_{1}$ and $T_{2}$ ) for $\gamma$ natural periods of vibration for $\gamma$ covering the interval [0.0001, 0.15 ] at various levels of $\tau$. Generally speaking, low values of $\tau$ (i.e., $\tau \leqslant 0.0001 \operatorname{secs}^{2}$ ) correspond to systems having a stiff superstructure. High values of $\tau$ (i.e., $\tau \geqslant 0.01$ secs $^{2}$ ) correspond to systems having a flexible superstructure. During a nonlinear time-history response, $\gamma$ will vary as the system switches between elastic and plastic states. In contrast, values of $\tau$ remain constant. Hence, from an analysis and design perspective, we need to explore sensitivity of parameters in the bang-bang control strategy to systematic variations in $\gamma$ while holding $\tau$ constant.

\subsection{Sensitivity analysis}

To understand the relative importance of displacement and velocity terms in the bang-bang control strategy, we employ a combination of mathematics and graphics to identify and validate trends in the "system velocity coefficients" versus $\xi, \gamma$ and $\tau$. First, notice that although each formula has many terms, the relationship among these coefficients is simple:

$$
\frac{B^{T} S(1,3)}{B^{T} S(1,4)}=1+\sqrt{\gamma} \text { and } \frac{B^{T} S(2,4)}{B^{T} S(1,4)}=1+\gamma+\sqrt{\gamma}
$$

For large values of $\gamma$ (e.g., $\gamma=0.15)$, the ratios $B^{T} S(1,3) / B^{T} S(1,4)$ and $B^{T} S(2,4) / B^{T} S(1,4)$ are 1.38 and 1.53 , respectively. For small values of $\gamma$ (i.e., $\gamma \sim 0.00$ ), the ratios $B^{T} S(1,3) / B^{T} S(1,4)$ and $B^{T} S(2,4) / B^{T} S(1,4)$ Approach 1. Hence, while the left-hand side of $\mathbf{B}^{\mathbf{T}} \mathbf{S}$ has coefficient values $1 / 2$ along the diagonal elements and zeros elsewhere, for small values of $\gamma$, values of the four system velocity components are approximately the same. Moreover, we note that:

$$
\operatorname{limit}_{\gamma \rightarrow 0}\left[\frac{\sqrt{(\gamma+2)-\sqrt{\gamma^{2}+4}}+\sqrt{(\gamma+2)+\sqrt{\gamma^{2}+4}}}{[4+2 \gamma+4 \sqrt{\gamma}]}\right]=\frac{1}{2} .
$$


$\left(B^{\top} S\right)_{1,3}$ vs. Change in Base Isolator Stiffness (Gamma) (tau=m/k)

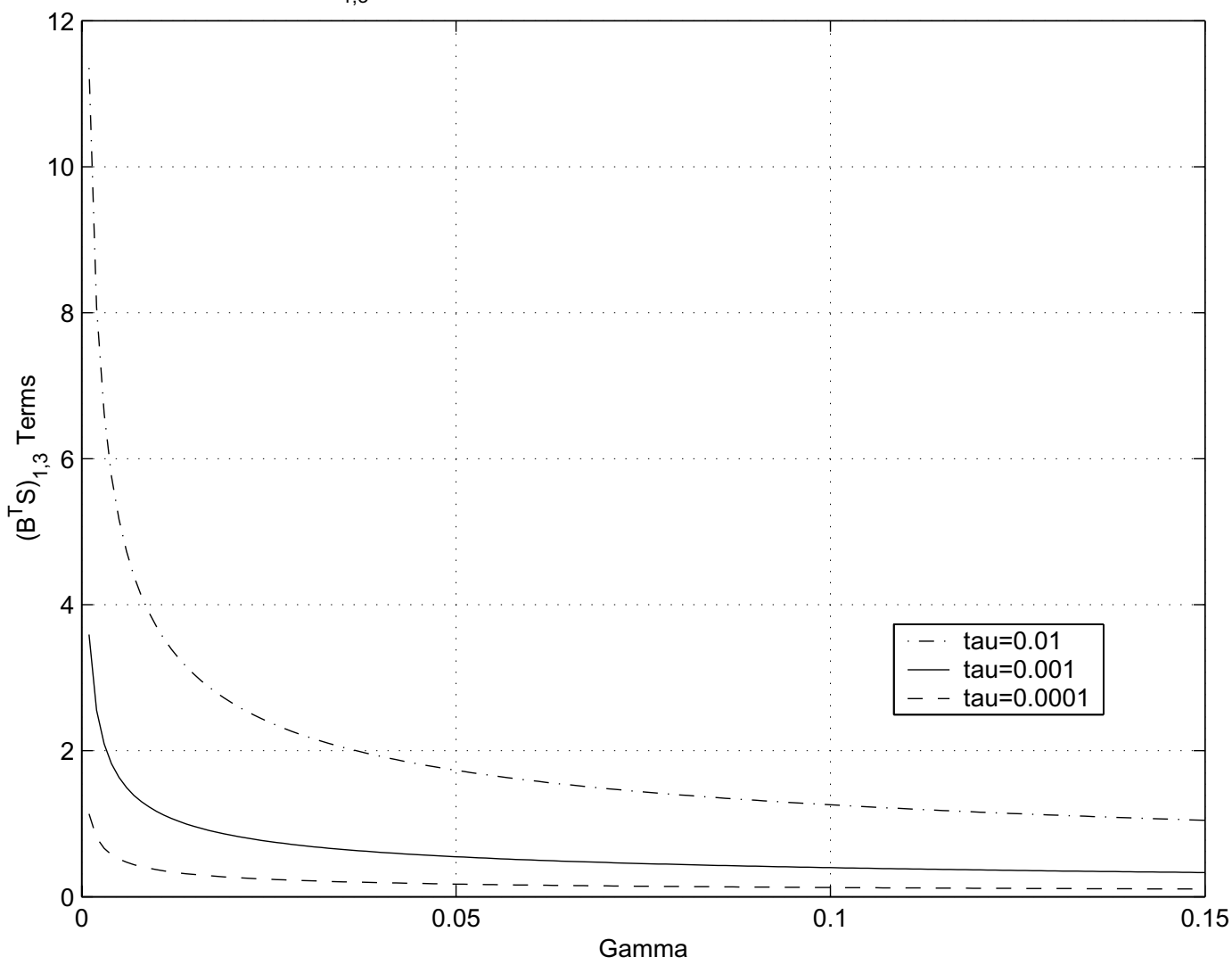

Fig. 2. Velocity coefficient $B^{T} S(1,3)$ versus $\gamma$ for contours of constant $\tau$.

Hence, for small values of $\gamma$, the velocity coefficients $B^{T} S(1,3), B^{T} S(1,4)$ and $B^{T} S(2,4)$ increase in magnitude in proportion to $1 / \sqrt{\gamma}$. Also, for a fixed value of $\gamma$, the velocity coefficients increase in proportion to $\sqrt{\tau}$ and $1 / \xi$ (the damping ratio of the structure).

Figures 2 through 4 validate these observations and show two important trends. First, we observe that $\tau$ increases monotonically as one moves vertically along contours of constant $\gamma$. We conclude from this trend that the influence of system velocities on bang-bang control will increase as the superstructure becomes progressively flexible. Conversely, bang-bang control will be most influenced by system displacements when the superstructure is stiff. Second, within the interval $\gamma \in[0.05,0.15]$, the coefficient values are relatively constant. We surmise from this observation that sub-optimal bang-bang control strategies will be insensitive to localized nonlinearities in the base isolation devices, especially when $\gamma(\mathrm{t})$ remains within the interval [0.05,0.15]. This observation also hints that for design applications where post-yield stiffnesses are very low (i.e., $\min (\gamma(t)) \approx 0$ ), the bang-bang control strategy is likely to switch between two modes: (1) a displacement driven strategy for pre-yield states, and (2) a velocity driven strategy for post-yield states.

\section{Summary and conclusions}

This paper has motivated the potential benefits in supplementing base isolation systems with sub-optimal bangbang control, and presented general symbolic analyses for one- and two-degree of freedom systems. The key benefit of symbolic analysis is improved insight into system functionality, particularly dependency mechanisms between the system state (characterized by displacements and velocities) and parameters (e.g., mass, stiffness, damping), and the 


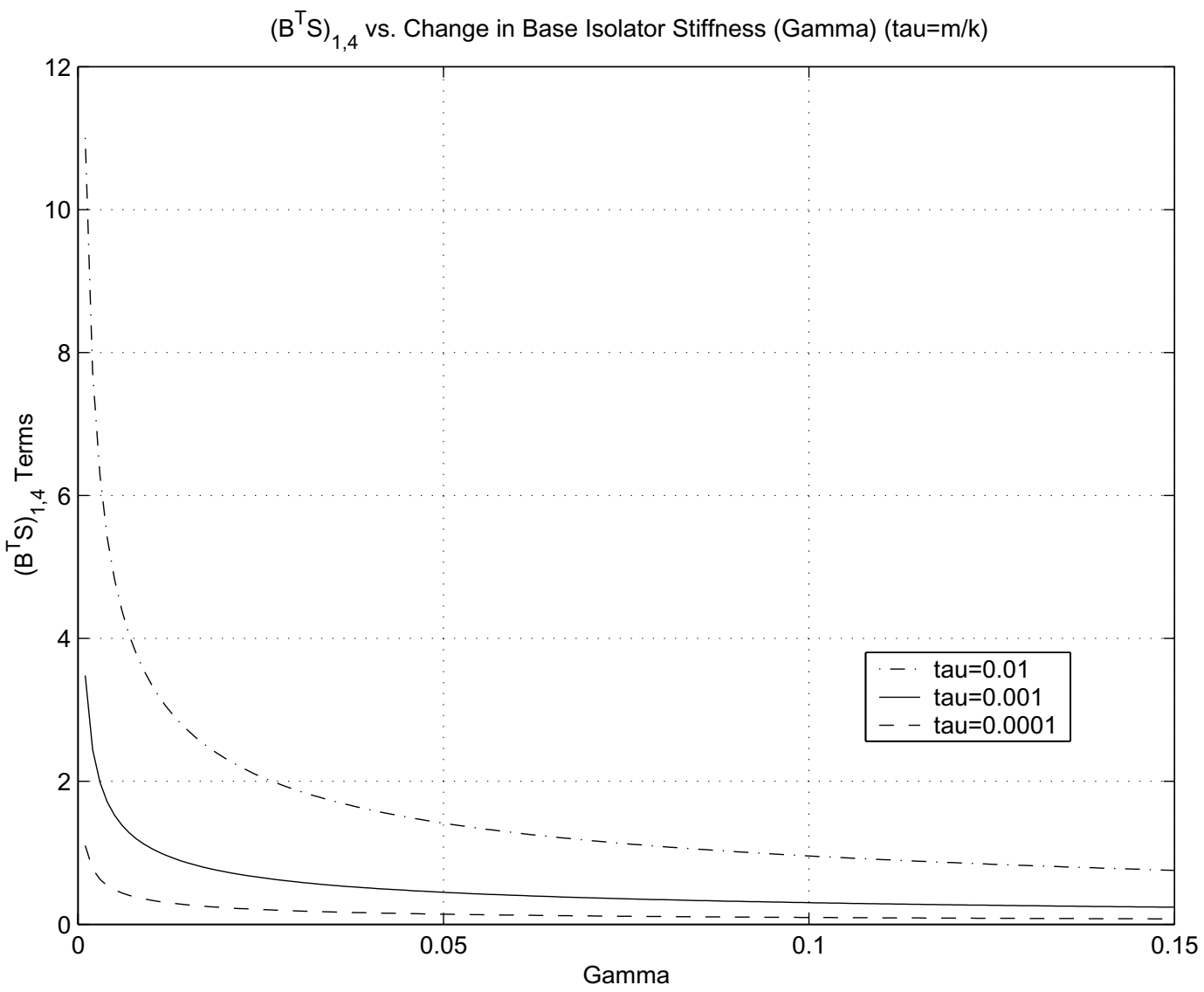

Fig. 3. Velocity coefficient $B^{T} S(1,4)$ versus $\gamma$ for contours of constant $\tau$.

sub-optimal bang-bang control strategy. The symbolic analyses suggest that sub-optimal bang-bang control strategies will be insensitive to localized nonlinearities in the base isolation devices, especially when $\gamma(\mathrm{t})$ remains within the interval [0.05,0.15]. As such, simplified design procedures might be justified. Unfortunately, symbolic analysis cannot determine the extent to which sub-optimal bang-bang control can actually improve the system performance. For estimates of the latter we still need to rely on linear/non-linear time history analyses. A computational framework for energy-balance and power-demand assessment of these system can be found in Sebastianelli and Austin [23].

\section{Appendix I. Scalable solutions to the Lyapanov matrix equation}

When the mass, damping and stiffness matrices are subject to the restrictions:

1. The mass matrix, $\mathbf{M}$, must be diagonal and uniform (i.e., $m_{1}=m_{2}=\ldots=m_{n}$ ).

2. Linear viscous damping (in the form $\alpha \cdot \mathbf{M}+\beta \cdot \mathbf{K}$ ) must be present in the system.

3. The structural stiffness matrix, $\mathbf{K}$, must be well-conditioned.

the symbolic solution to the Lyapunov matrix equation for a 1-DOF system is scalable to an $n$-DOF system.

Proof: Consider a 1-DOF system with stiffness, $k$, mass, $m$, and linear viscous damping, $c=\alpha \cdot m+\beta \cdot k$. Symbolic solutions to $\mathbf{B}^{\mathbf{T}} \mathbf{S}$ are as given in Eqs (15), (17) and (18). Substituting the definition for $\mathbf{A}-$ for details, see Eq. (3) - into the matrix equation $\mathbf{A}^{\mathbf{T}} \mathbf{S}+\mathbf{S A}=-\mathbf{Q}$, and noting that $\mathbf{K}^{\mathbf{T}}=\mathbf{K}$ and $\mathbf{M}^{\mathbf{T}}=\mathbf{M}$ leads to:

$$
\mathbf{A}^{\mathbf{T}} \mathbf{S}+\mathbf{S A}=\left[\begin{array}{c}
\mathbf{0}-\left([\mathbf{K}]\left[\mathbf{M}^{-\mathbf{1}}\right]\right) \\
{[\mathbf{I}]-\left([\mathbf{C}]\left[\mathbf{M}^{-\mathbf{1}}\right]\right)}
\end{array}\right] \mathbf{S}+\mathbf{S}\left[\begin{array}{cc}
\mathbf{0} & {[\mathbf{I}]} \\
-\left(\left[\mathbf{M}^{-\mathbf{1}}\right][\mathbf{K}]\right)-\left(\left[\mathbf{M}^{-\mathbf{1}}\right][\mathbf{C}]\right)
\end{array}\right]=-\mathbf{Q} .
$$


$\left(B^{\top} S\right)_{2,4}$ vs. Change in Base Isolator Stiffness (Gamma) (tau=m/k)

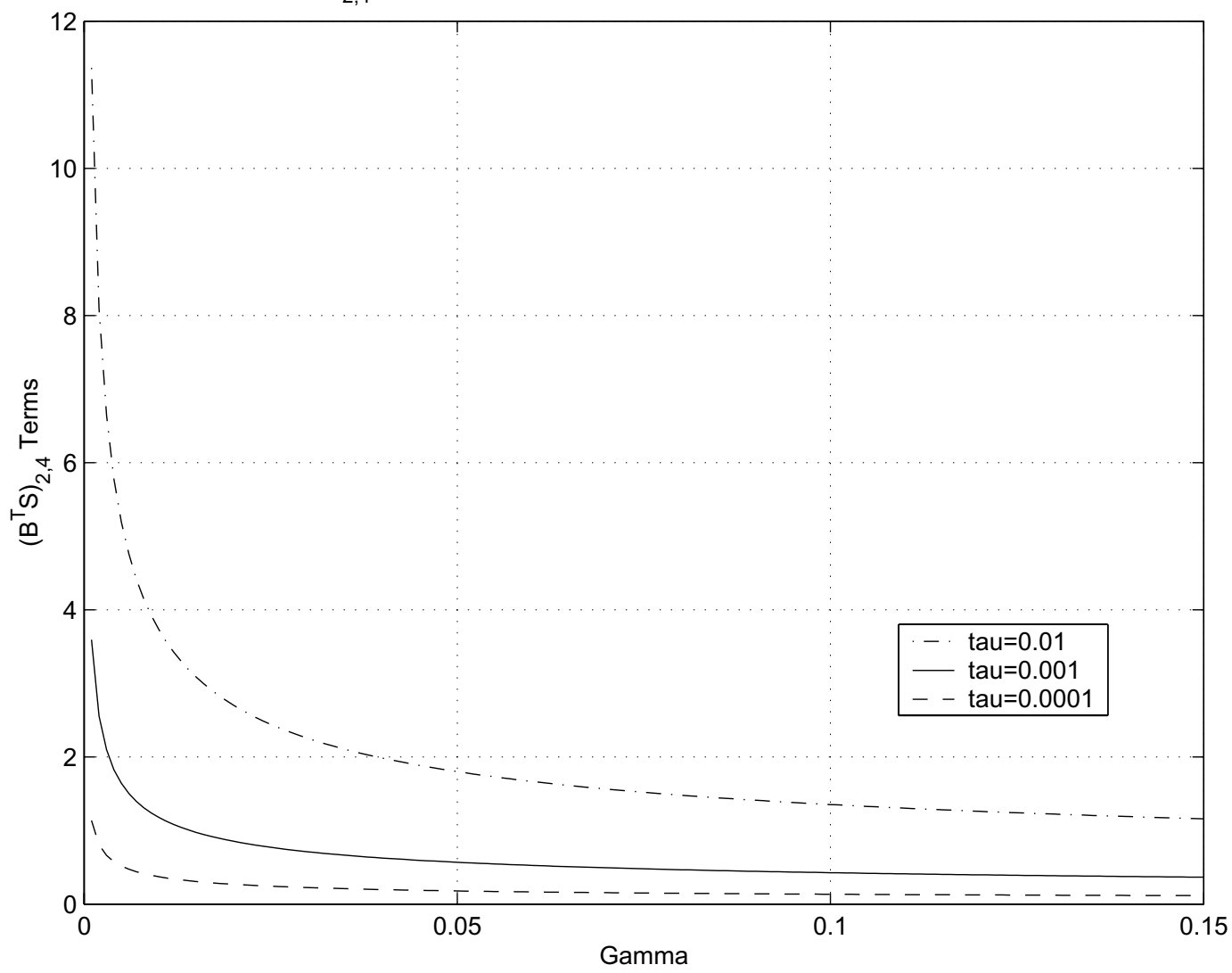

Fig. 4. Velocity coefficient $B^{T} S(2,4)$ versus $\gamma$ for contours of constant $\tau$.

Now notice that the matrix elements of Eq. (53) are symbolic expressions expressed as fractions. For the matrix counterpart of Eqs (15), (17) and (18) to exist, one can either pre- or post-multiply the matrix expression appearing as the denominator of each matrix element. We investigate both scenarios to ascertain if, and under what conditions, the symbolic form for the SDOF system will scale to a N-DOF system.

\section{Pre-Multiplication for Matrix Product $\mathbf{A}^{\mathrm{T}} \mathbf{S}$ :}

$$
\begin{aligned}
& \text { Element 1-1 }=-\frac{1}{2}\left[\mathbf{K}^{-\mathbf{1}}\right][\mathbf{K}]\left[\mathbf{K}^{\star}\right]=-\frac{1}{2}\left[\mathbf{K}^{\star}\right] \\
& \text { Element 1-2 }=-\frac{1}{2}[\mathbf{K}]\left[\mathbf{M}^{-\mathbf{1}}\right]\left[\mathbf{K}^{-\mathbf{1}}\right]\left[\mathbf{C}^{-\mathbf{1}}\right][\mathbf{M}][\mathbf{M}]\left[\mathbf{K}^{\star}\right] \\
& \text { Element 2-1 }=\frac{1}{2}\left[\mathbf{C}^{-\mathbf{1}}\right][\mathbf{M}]\left[\mathbf{K}^{\star}\right]+\frac{1}{2}\left[\mathbf{K}^{-\mathbf{1}}\right][\mathbf{C}]\left[\mathbf{K}^{\star}\right]-\frac{1}{2}[\mathbf{C}]\left[\mathbf{M}^{-\mathbf{1}}\right]\left[\mathbf{K}^{-\mathbf{1}}\right][\mathbf{M}]\left[\mathbf{K}^{\star}\right] \\
& \text { Element 2-2 }=\frac{1}{2}\left[\mathbf{K}^{-\mathbf{1}}\right][\mathbf{M}]\left[\mathbf{K}^{\star}\right]-\frac{1}{2}[\mathbf{C}]\left[\mathbf{M}^{-\mathbf{1}}\right]\left[\mathbf{K}^{-\mathbf{1}}\right]\left[\mathbf{C}^{-\mathbf{1}}\right][\mathbf{M}][\mathbf{M}]\left[\mathbf{K}^{\star}\right]
\end{aligned}
$$

Pre-multiplication for matrix product $\mathbf{S A}$ :

$$
\text { Element 1-1 }=-\frac{1}{2}\left[\mathbf{K}^{-\mathbf{1}}\right][\mathbf{M}]\left[\mathbf{K}^{\star}\right]\left[\mathbf{M}^{-\mathbf{1}}\right][\mathbf{K}]
$$




$$
\begin{aligned}
& \text { Element 1-2 }=\frac{1}{2}\left[\mathbf{C}^{-\mathbf{1}}\right][\mathbf{M}]\left[\mathbf{K}^{\star}\right]+\frac{1}{2}\left[\mathbf{K}^{-\mathbf{1}}\right][\mathbf{C}]\left[\mathbf{K}^{\star}\right]-\frac{1}{2}\left[\mathbf{K}^{-\mathbf{1}}\right][\mathbf{M}]\left[\mathbf{K}^{\star}\right][\mathbf{C}]\left[\mathbf{M}^{-\mathbf{1}}\right] \\
& \text { Element 2-1 }=-\frac{1}{2}\left[\mathbf{K}^{-\mathbf{1}}\right]\left[\mathbf{C}^{-\mathbf{1}}\right][\mathbf{M}][\mathbf{M}]\left[\mathbf{K}^{\star}\right][\mathbf{K}]\left[\mathbf{M}^{-\mathbf{1}}\right] \\
& \text { Element 2-2 }=\frac{1}{2}\left[\mathbf{K}^{-\mathbf{1}}\right][\mathbf{M}]\left[\mathbf{K}^{\star}\right]-\frac{1}{2}\left[\mathbf{K}^{-\mathbf{1}}\right]\left[\mathbf{C}^{-\mathbf{1}}\right][\mathbf{M}][\mathbf{M}]\left[\mathbf{K}^{\star}\right][\mathbf{C}]\left[\mathbf{M}^{-\mathbf{1}}\right]
\end{aligned}
$$

When $\left[\mathbf{K}^{\star}\right]=[\mathbf{K}]$ and $[\mathbf{M}]$ is diagonal and uniform (i.e., $m_{1}=m_{2}=\ldots=m_{n}$ ), Eqs (54) through (61) simplify to:

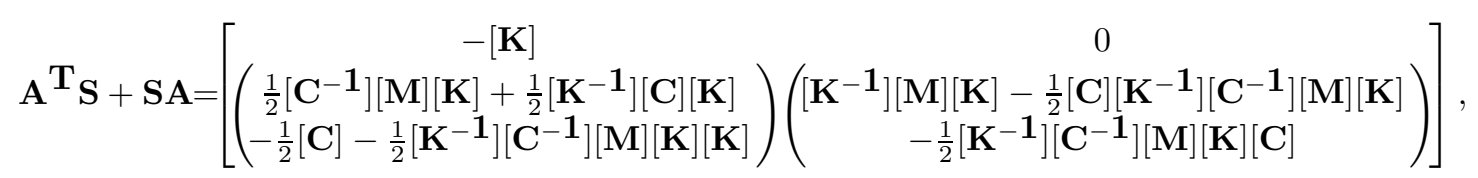

Note the following equalities:

1. $\left[\mathbf{K}^{-1}\right][\mathbf{C}][\mathbf{K}]=[\mathbf{C}]$

2. $\left[\mathrm{K}^{-\mathbf{1}}\right]\left[\mathrm{C}^{-\mathbf{1}}\right][\mathrm{M}][\mathrm{K}]=\left[\mathrm{C}^{-\mathbf{1}}\right][\mathrm{M}]$

3. $[\mathbf{C}]\left[\mathbf{K}^{-1}\right]\left[\mathbf{C}^{-1}\right]=\left[\mathbf{K}^{-\mathbf{1}}\right]$

4. $\left[\mathbf{C}^{-1}\right][\mathbf{M}][\mathbf{K}][\mathbf{C}]=[\mathbf{M}][\mathbf{K}]$

Substituting these equalities into Eq. (62) and simplifying terms gives:

$$
\mathbf{A}^{\mathbf{T}} \mathbf{S}+\mathbf{S A}=\left[\begin{array}{cc}
-[\mathbf{K}] & \mathbf{0} \\
\mathbf{0} & \mathbf{0}
\end{array}\right]=-\mathbf{Q}
$$

\section{Post-Multiplication Matrix Strategy for S}

Repeating this computation for post-multiplication leads to:

$$
\mathbf{A}^{\mathbf{T}} \mathbf{S}+\mathbf{S A}=\left[\begin{array}{cc}
-[\mathbf{K}] & -\frac{1}{2}[\mathbf{K}][\mathbf{M}]\left[\mathbf{C}^{-\mathbf{1}}\right]+\frac{1}{2}[\mathbf{M}][\mathbf{K}]\left[\mathbf{C}^{-\mathbf{1}}\right] \\
\frac{1}{2}[\mathbf{M}][\mathbf{K}]\left[\mathbf{C}^{-\mathbf{1}}\right]-\frac{1}{2}[\mathbf{M}]\left[\mathbf{C}^{-\mathbf{1}}\right][\mathbf{K}] & \frac{1}{2}[\mathbf{M}]-\frac{1}{2}[\mathbf{C}][\mathbf{M}]\left[\mathbf{C}^{-\mathbf{1}}\right]
\end{array}\right] .
$$

Note the following equalities:

1. $[\mathbf{K}]\left[\mathbf{C}^{-\mathbf{1}}\right]=\left([\mathbf{K}]\left[\mathbf{C}^{-\mathbf{1}}\right]\right)^{\mathbf{T}}=[\mathbf{C}]^{\mathbf{T}}[\mathbf{K}]^{\mathbf{T}}=\left[\mathbf{C}^{-\mathbf{1}}\right][\mathbf{K}]$

2. $[\mathbf{C}][\mathbf{M}]\left[\mathbf{C}^{-\mathbf{1}}\right]=[\mathbf{M}]$

3. $[\mathbf{K}][\mathbf{M}]=([\mathbf{K}][\mathbf{M}])^{\mathbf{T}}=[\mathbf{M}]^{\mathbf{T}}[\mathbf{K}]^{\mathbf{T}}=[\mathbf{M}][\mathbf{K}]$

Substituting these equalities into Eq. (64) and simplifying terms gives:

$$
\mathbf{A}^{\mathbf{T}} \mathbf{S}+\mathbf{S A}=\left[\begin{array}{cc}
-[\mathbf{K}] & \mathbf{0} \\
\mathbf{0} & \mathbf{0}
\end{array}\right]=-\mathbf{Q}
$$

\section{References}

[1] AASHTO, Guide Specifications for Seismic Isolation Design, 1991.

[2] S. Adhikari, Damping Models for Structural Vibration, PhD thesis, Cambridge University, September 2000.

[3] T. Andriono and A.J. Carr, Reduction and Distribution of Lateral Seismic Forces on Base Isolated Mutli-Storey Structures, Bulletin of the New Zealand National Society for Earthquake Engineering 24(3) (September 1991), 225-237.

[4] M.A. Austin and W.J. Lin, Energy-Balance Assessment of Isolated Structures, Journal of Engineering Mechanics, ASCE 130(3) (2004), $347-358$.

[5] M.A. Austin and R. Sebastianelli, Phase Analysis of Actuator Response for Sub-Optimal Bang-Bang and Velocity Cancellation Control of Base Isolated Structures, Journal of Structural Control and Health Monitoring 14(7) (2007), 1034-1061. 
[6] M.A. Austin, K.S. Pister and S.A. Mahin, A Methodology for the Probabilistic Limit States Design of Earthquake Resistant Structures, Journal of the Structural Division, ASCE 113(8) (August 1987), 1642-1659.

[7] R. Bellman, I. Glicksberg and O. Gross, On the Bang-Bang Control Problem, Quarterly of Applied Mathematics 14(1) (1956), 11-18.

[8] R.W. Clough and J. Penzien, Dynamics of Structures, McGraw-Hill, New York, New York, 1993.

[9] A. Ghobarah and H.M. Ali, Seismic Design of Base-Isolated Highway Bridges utilizing Lead-Rubber Bearings, Canadian Journal of Civil Engineering 17 (1990), 413-422.

[10] J.F. Hall, T.H. Heaton, M.W. Halling and D.J. Wald, Near-Source Ground Motion and its Effects on Flexible Buildings, Earthquake Spectra 11(4) (1995), 569-605

[11] T.H. Heaton, J.F. Hall, D.J. Wald and M.W. Halling, Response of High-Rise and Base-Isolated Buildings in a Hypothetical Mw 7.0 Blind Thrust Earthquake, Science, 267.

[12] J. Inaudi, F. Lopez-Almansa, J.M. Kelly and J. Rodellar, Predictive Control of Base Isolated Structures, Earthquake Engineering and Structural Dynamics 21 (1992), 471-482.

[13] International Conference of Building Officials (ICBO). Uniform Building Code Regulation for Seismic-Isolated Structures, 1997, Volume 2, Appendix Chapter 16.

[14] L.S. Jacobson, Steady Forced Vibrations as Influenced by Damping, Transactions, ASME APM-52-13, 1930, pages 169-181.

[15] E.A. Johnson, J.C. Ramallo, B.F. Spencer and M.F. Sain, Intelligent Base Isolation Systems, In Proceedings of Second World Conference on Structural Control, Kyoto, Japan, 1998

[16] T. Kailath, Linear Systems, Prentice-Hall, London, England, 1980.

[17] R.K. Miller, T.J. Dehghanyar and T.K. Caughey, Active Vibration Control of Large Civil Structures, Journal of Engineering Mechanics, ASCE 114(9) (September 1988).

[18] P.J. Moss and A.J. Carr, Structural Damping Models for Non-linear Seismic Analysis, in: Proceedings of New Zealand Society of Earthquake Engineering, Taupo, New Zealand, March 1994.

[19] F. Naeim and J. Kelly, Design of Seismic Isolated Structures, John Wiley and Sons, 1998.

[20] N.N. Newmark and W.J. Hall, Earthquake Spectra and Design. A Primer, Earthquake Research Institute, Berkeley, CA, 1982.

[21] J.C. Ramallo, E.A. Johnson, B.F. Spencer and M.K. Sain, SemiActive Building Base Isolation, In Proceedings of the American Control Conference, San Diego, California, June 1999.

[22] Rayleigh, Lord, Theory of Sound, (2nd Edition) Dover Publications, 1945.

[23] R. Sebastianelli and M.A. Austin, Energy Balance and Power Demand Assessment of Actuators in Base Isolated Structures supplemented with Modified Bang-Bang Control, Structural Engineering and Mechanics 25(5) (March 2007), 541-564.

[24] B.F. Spencer and S. Nagarajaiah, State of the Art of Structural Control, Journal of Structural Engineering, ASCE, July 2003, pages $845-856$.

[25] H.-C. Tsai and J.M. Kelly, Non-classical Damping in Dynamic Analysis of Base Isolated Structures with Internal Equipment, Earthquake Engineering and Structural Dynamics 16(1) (1988), 29-43.

[26] D.H. Turkington, A.J. Carr, N. Cooke and P.J. Moss, Design Method for Bridges on Lead-Rubber Bearings, Journal of the Structural Division, ASCE 115(12) (December 1989), 3017-3030.

[27] D.H. Turkington, A.J. Carr, N. Cooke and P.J. Moss, Seismic Design of Bridges on Lead-Rubber Bearings, Journal of the Structural Division, ASCE 115(12) (December 1989), 3000-3016.

[28] S. Wolfram, The Mathematica Book, Cambridge University Press, Cambridge, MA, 1999.

[29] W.M. Wonham and C.D. Johnson, Optimal Bang-Bang Control with Quadratic Performance Index, Journal of Basic Engineering 86 (1964), 107-115.

[30] Z. Wu and T.T. Soong, Modified Bang-Bang Control Law for Structural Control Implementation, Journal of Engineering Mechanics, ASCE 122(8) (1996), 771-777.

[31] H. Yoshioka, J.C. Ramallo and B.F. Spencer, Jr., Smart Base Isolation Strategies Employing Magnetorheological Dampers, Journal of Engineering Mechanics 128(5) (2002), 540-551. 

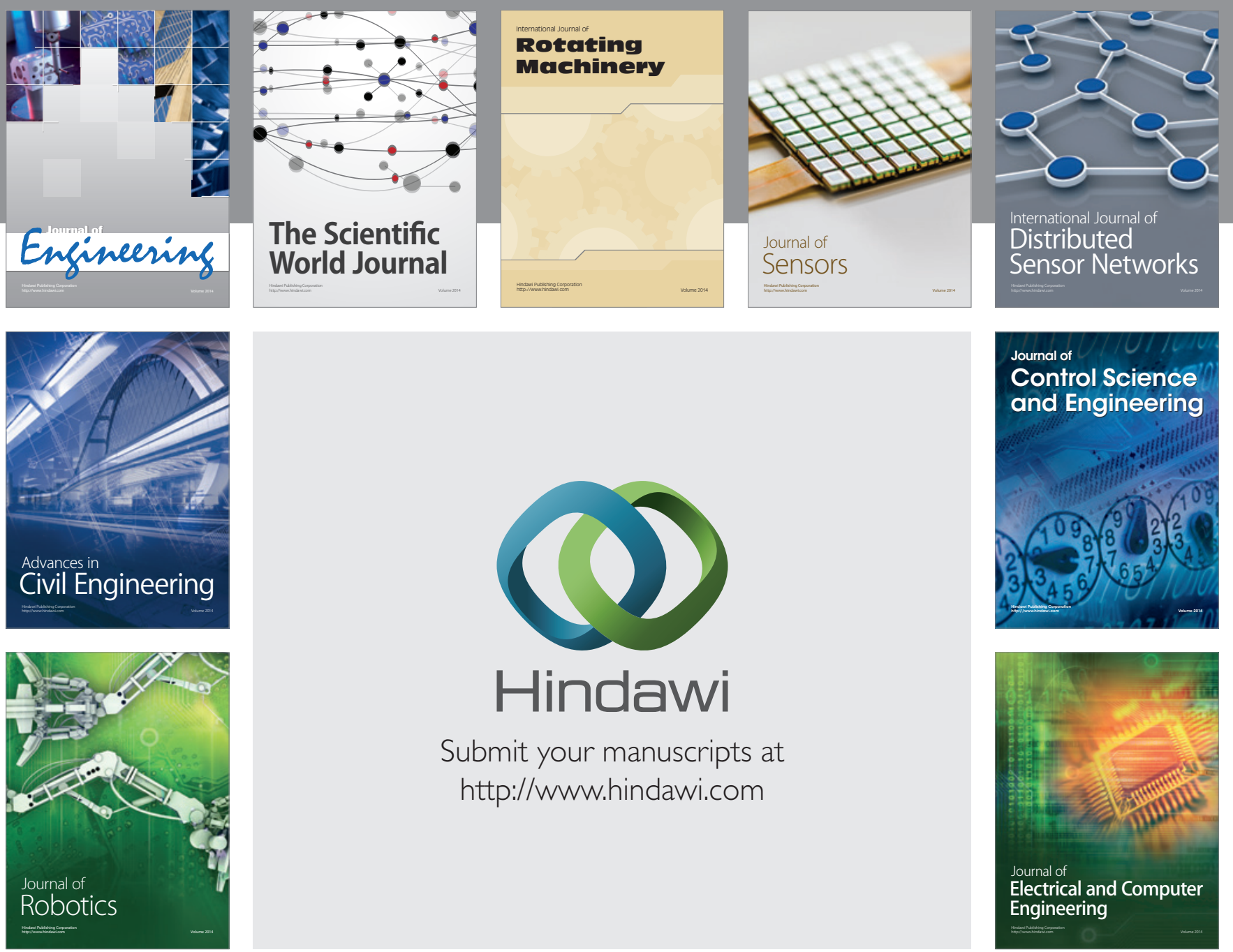

Submit your manuscripts at

http://www.hindawi.com
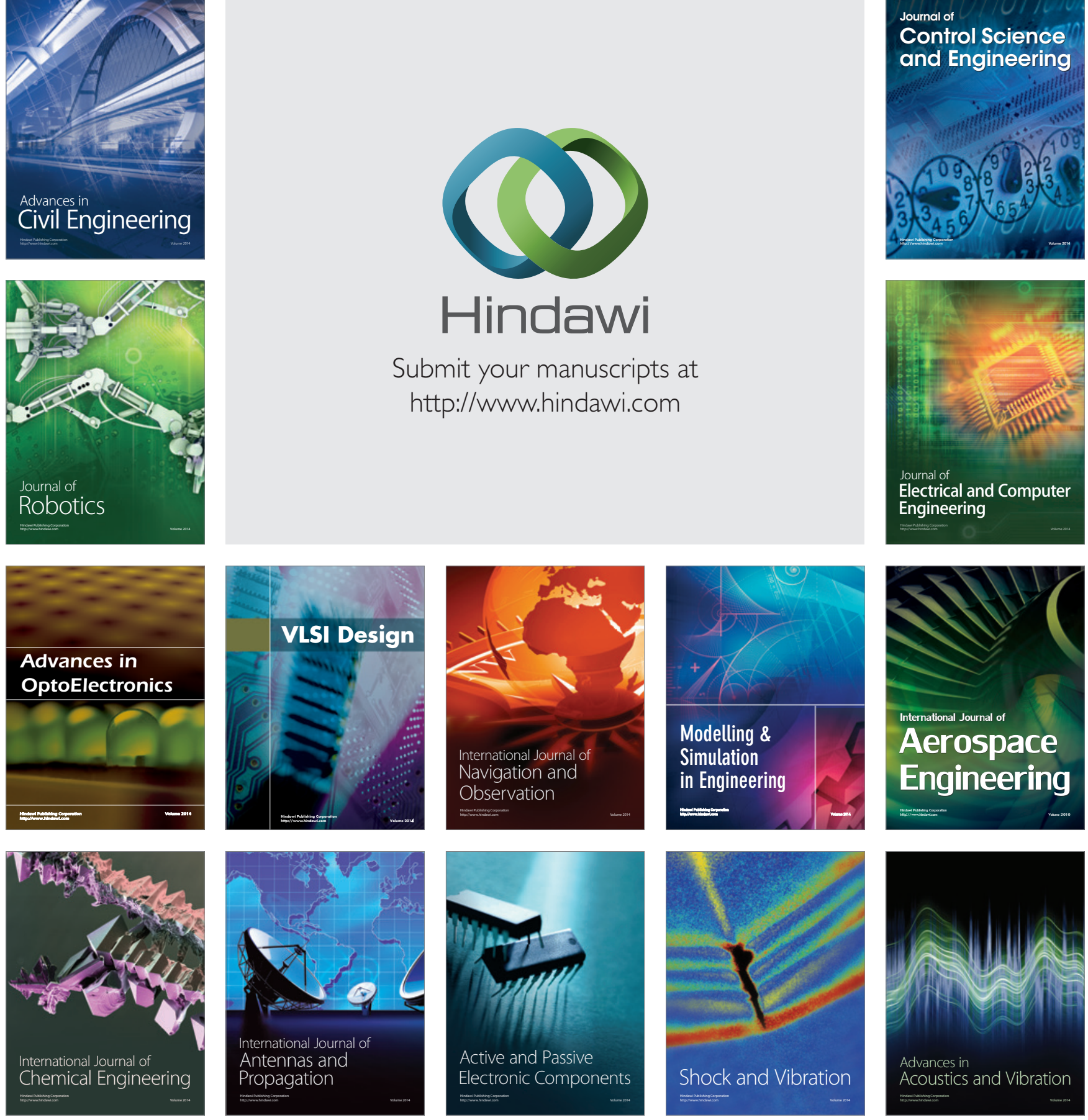